\title{
Study of II Galactic quadrant of Milky Way Galaxy using open clusters
}

\author{
Devendra Bisht ${ }^{1 *}$, Shashikiran Ganesh ${ }^{1 \dagger}$, Kiran Singh Baliyan ${ }^{1 \ddagger}$, \\ Ramakant Singh Yadav ${ }^{2 \S}$, Alok Durgapal ${ }^{3 \pi}$
}

\author{
${ }^{1}$ Astronomy and Astrophysics Division, Physical Research Laboratory, Ahmedabad, India, 380009 \\ ${ }^{2}$ Aryabhatta Research Institute of observational sciences, Manora peak, Nainital, India \\ ${ }^{3}$ Department of physics, DSB campus, Kumaun University Nainital, India, 263002
}

\begin{abstract}
We have made $U B V I$ CCD observations for the open clusters Teutsch 1, Riddle 4 and Czernik 6 using 1.04-m Sampurnanand telescope located at the ARIES observatory (Manora peak, Nainital, India). We have used $2 M A S S J H K_{s}$ data for the clusters Teutsch 126, Teutsch 54 and Czernik 3. For the estimation of fundamental parameters, we have plotted radial density profiles, colour-magnitude and colour-colour diagrams. Using these inputs, we have studied the structure of Milky Way Galaxy in the second Galactic quadrant. We have considered the open clusters that are younger than 1 Gyrs and lay in the longitude range from 90 to 180 deg. Our study shows that up to $3.5 \mathrm{Kpc}$, the Galactic disc bends towards the southern hemisphere while after $3.5 \mathrm{Kpc}$ it bends towards the northern hemisphere. The distribution of reddening with longitude and age shows a decreasing trend with the longitude and age of the clusters. Our study also indicates that younger clusters have more reddening than older ones.
\end{abstract}

\section{Introduction}

Open star clusters are the best test areas for stellar evolution, Galactic structure and chemical evolution of the Galactic disc. Becker (1963) pointed out the similarity between the distributions of young open clusters in our galaxy. Since then, many contributions have aimed at getting a better picture of our Galactic spiral arms by adding more young clusters to the material and by including other types of objects.

This paper discusses the structure of Milky Way Galaxy and its spiral arms by using open clusters. The distribution of star clusters in the longitude range $l=90-180 \mathrm{deg}$ shows a slight bend of the Galactic disc in the southern hemisphere within $3.5 \mathrm{kpc}$ distance. After $3.5 \mathrm{kpc}$, the distribution indicates a bend towards the northern hemisphere. Solomon et al. (1987) traced a face-on picture of warm molecular clouds in the first Galactic quadrant ( $l$ between 8 and 90 deg.), delineating three

\footnotetext{
*dbisht@prl.res.in

†shashi@prl.res.in

†baliyanprl@gmail.com

§rkant@aries.res.in

ףalokdurgapal@gmail.com
} 


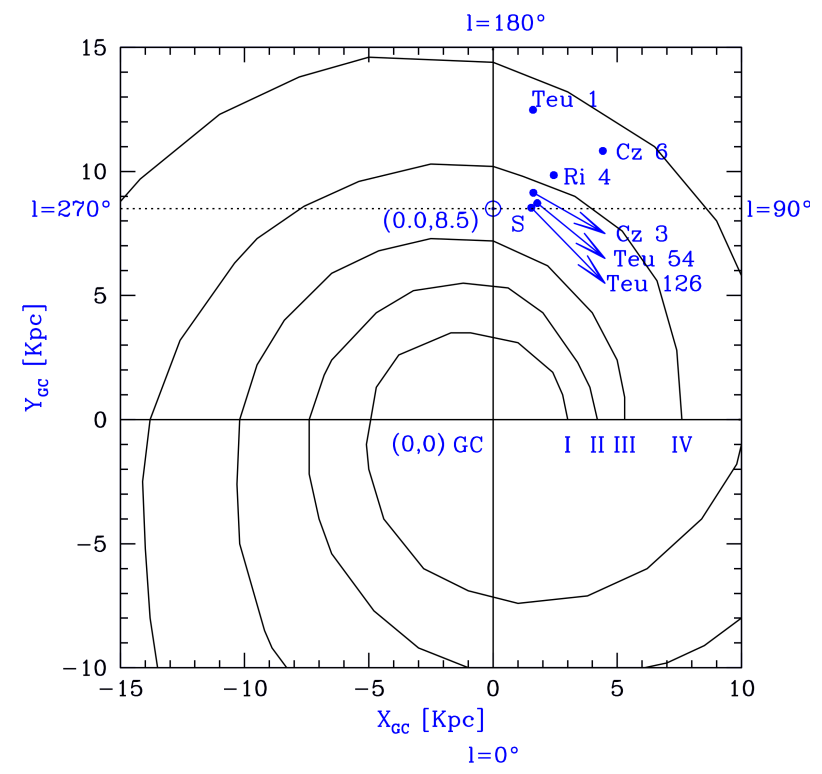

Figure 1: Schematic view of the spiral structure of the Galaxy (Valee et al. 2005). $X_{G C}$ indicates the direction of the Galactic rotation, and $Y_{G C}$ represents the distance to the anti-center. The position of the Sun $(0.0,8.5)$ and the Galactic center $(0.0,0.0)$ are indicated by S and GC respectively. The symbols I, II, III and IV indicate the Norma (Outer), Scutum-Crux, Sagittarius, and Perseus arms, respectively.

arm-like structures. Kulkarni et al. (1982) identified the Perseus arms from $l=65$ to 150 deg and the Cygnus arm between $l=30$ to $110 \mathrm{deg}$. Heyer \& Terebey (1998) identified small and compact clouds beyond the Perseus arm and Digel et al. $(1994,1996)$ demonstrated the presence of molecular material within the outer Galaxy.

\section{Data Used}

The CCD optical data for the clusters Teutsch (Teu) 1, Czernik (Cz) 6, and Riddle (Ri) 4 were obtained in the $U B V I$ bands using the 1.04-m Sampurnanand telescope located at the ARIES observatory (Manora peak, Nainital, India). Data reduction and analysis for these clusters are explained in Bisht et al. (2016). We have used the $2 M A S S$ point source catalog data for the clusters Teu 61, Teu 126, and Cz 3. 2MASS (Skrutskie et al. 2006) uses two highly automated 1.3-m telescopes (one at Mt. Hopkins, Arizona (AZ), USA and another at the Cerro Tololo Inter-American Observatory, Chile) with a 3-channel camera, each channel having a $(256 \times 256)$ array of $\mathrm{HgCdTe}$ detectors. The $2 M A S S$ photometric catalog provides $J(1.25 \mu \mathrm{m}), H(1.65 \mu \mathrm{m})$, and $K s(2.17 \mu \mathrm{m})$ band photometry for millions of galaxies and nearly half a billion of stars (Carpenter 2001). The sensitivity of the $2 M A S S$ catalog is $15.8 \mathrm{mag}$ for $J, 15.1 \mathrm{mag}$ for $H$, and $14.3 \mathrm{mag}$ for $K s$ at a signal-to-noise of 10 . Bisht et al. (2017) have estimated the fundamental parameters for the clusters Teu 126, Teu 61, and Cz 3.

\section{Structure of Galactic disc between $l=90 \mathrm{deg}$ and $180 \mathrm{deg}$}

Open star clusters considered in the present study are located in the second Galactic quadrant and can be used to understand the Galactic disc structure in the direction of their location. The schematic view of the spiral structure of Milky Way Galaxy is shown in Fig. 1. $X$ and $Y$ represents the direction 
of Galactic rotation and distance to the anti-center, respectively. The position of the Sun is $(0.0,8.5)$ while the Galactic center is at $(0,0)$. The location of these star clusters indicates that $\mathrm{Ri} 4, \mathrm{Cz} 3$, Teu 54 and Teu 126 are in the nearby Perseus arm while Teu 1 and $\mathrm{Cz} 6$ may be part of the Outer arm.

To investigate the Galactic disc in the direction of these clusters, we have selected the star clusters from the WEBDA ${ }^{1}$ database, which exist between $l=90$ to $l=180 \mathrm{deg}$. These selected clusters (Dias et al. 2002) are younger than 1 Gyrs since young clusters do not move very far from the Galactic disc. All the selected clusters (Teu 1, Ri 4, Cz 6, Teu 126, Teu 54, and Cz 3) are plotted in Fig. 2. The open clusters $\mathrm{Ri}$ 4, Teu 1, Cz 6, Teu 126, Teu 54, and Cz 3 are indicated with a filled circle while others are shown by an open circle. Fig. 2(a) shows the distribution of the longitude and distance from the plane $Z$ while 2(b) shows the distribution of their heliocentric distance $D$ and $Z$. Both the distributions show that $45 \%$ of the clusters are above the Galactic plane while $55 \%$ are below. Teu $1, \mathrm{Cz} 6$, and Teu 54 are above and $\mathrm{Ri} 4$, Teu 126, and $\mathrm{Cz} 3$ are below the Galactic plane. The cluster's distribution indicates that the Galactic disc is inclined to the southern Galactic hemisphere in the studied longitude range. A similar feature has also been observed by Subramaniam et al. (2007) in the longitude range $l=90-135$ deg in the Galaxy. Fig. 2(b) shows two groups of clusters: one group contains clusters with distances less than $3.5 \mathrm{Kpc}$ and the second group contains clusters having a distance exceeding 3.5 Kpc. In the first group (including Ri 4, Teu 126, and Cz 3), the majority of the clusters are below the Galactic plane while in the second group (including Teu 1, Cz 6, and Teu 54), the clusters are above the Galactic plane. The location of the clusters belonging to first and second group can be considered as objects in the Perseus and in the outer arm of the Milky Way, respectively. Pandey et al. (2006) have shown that the outer arm is located at longitudes between $l \sim 120$ to $\sim 235 \mathrm{deg}$.

This kind of slight divergence in the Galactic disc may be considered due to a mild warp in the Galactic disc. Galactic warp has also been observed by Momany et al. (2006) based on the red clump stars located between 2 to $4 \mathrm{Kpc}$ from the Sun. They conclude that the Galactic disc shows a marginal bend towards negative latitudes. The clusters in the first group in Fig. 2(b) might be showing the same bend in a similar distance range. Russeil et al. (2003) have shown that the Norma-Cygnus (outer) arm exhibits a clear positive warp between $l=60-220 \mathrm{deg}$. Our second group of objects in Fig. 2(b) might be showing the same warp as seen in Russeil et al. (2003).

In Fig. 2(c) we plot the reddening versus the longitude of the clusters. This figure shows that reddening decreases with increasing longitudes. The estimated reddening for the clusters Teu $1, \operatorname{Ri} 4$ and $\mathrm{Cz} 6$ follows the same trend. Fig. 2(d) shows the distribution of the reddening of the clusters with their age. This distribution indicates that the reddening decreases with increasing age of the cluster.

\section{Dependence of mass function slope with other parameters}

The distribution of the stellar masses at birth is defined as the mass function (MF). The mass function for massive stars $\left(\geq 1 M_{\odot}\right)$ has been studied and well established by Salpeter (1955). We have used six open clusters (Teu 1, Ri 4, Cz 6, Cz 3, Teu 126, and Teu 54) to see the dependence of the slope of the MF on cluster age, core radius $\left(R_{c}\right)$, galactocentric distance $\left(R_{G C}\right)$, and heliocentric distance $(D)$ as shown in Fig. 3. Using the following relation, we have estimated the galactocentric distance

$$
\mathrm{R}_{\mathrm{GC}}=\sqrt{(\mathrm{D} \times \cos (\mathrm{b}))^{2}+8.5^{2}-2 \times \mathrm{D} \times 8.5 \times \cos (\mathrm{l}) \times \cos (\mathrm{b})}
$$

where $l$ and $b$ are the longitude and latitude of the cluster. We determined the average value of the MF slope as $1.55 \pm 0.4$ for above $1 M_{\odot}$. We find that the MF slope does not show any trend with these parameters. However, more data points are required to confirm it.

\footnotetext{
${ }^{1}$ http://obswww.unige.ch/webda
} 

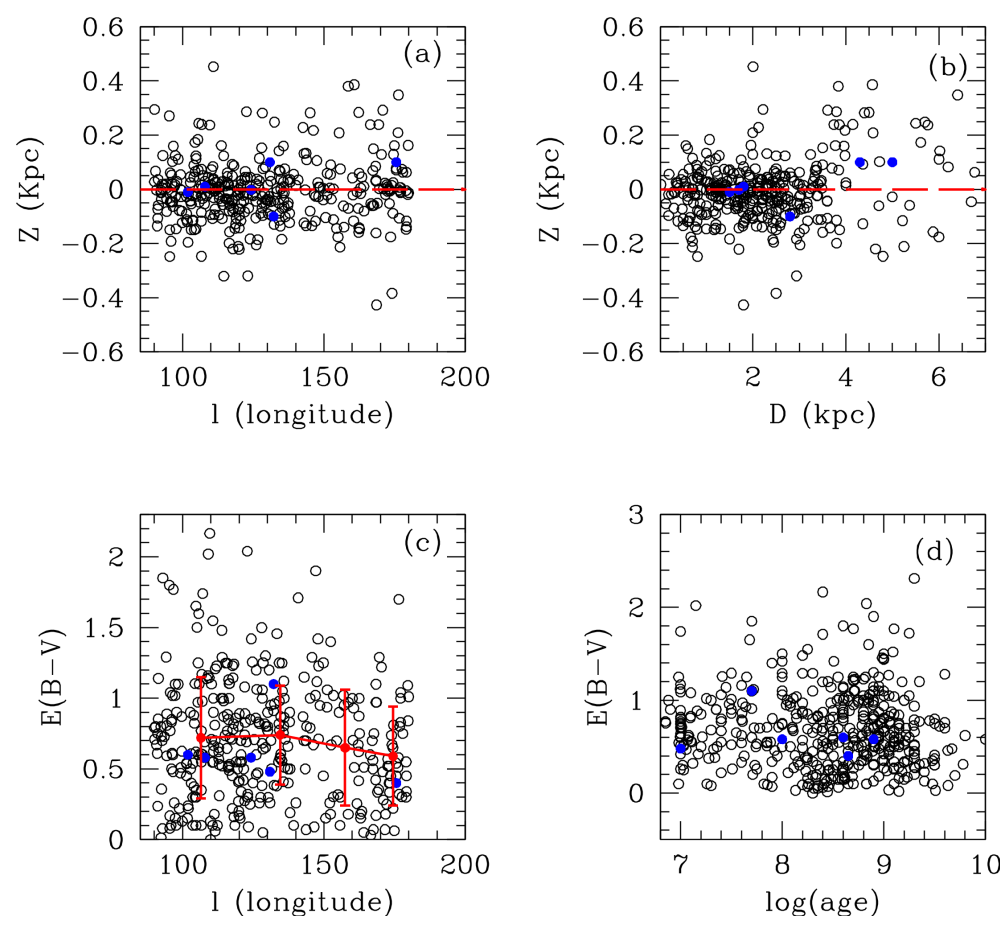

Figure 2: Distributions of star clusters with $l=90-180 \mathrm{deg}$, distances more than $2 \mathrm{Kpc}$, and ages below than 1 Gyrs. (a) Distribution of the Galactic height $(Z)$ and longitude $(l)$. (b) Distribution of $Z$ and heliocentric distance $(D)$. (c) Distribution of reddening $(E(B-V))$ and longitude. (d) Distribution of $E(B-V)$ and clusters age. In this figure blue dots are our observed clusters, while black dots are the clusters taken from Dias et al. (2002). The red solid line with error bar indicates the average value of interstellar reddening.

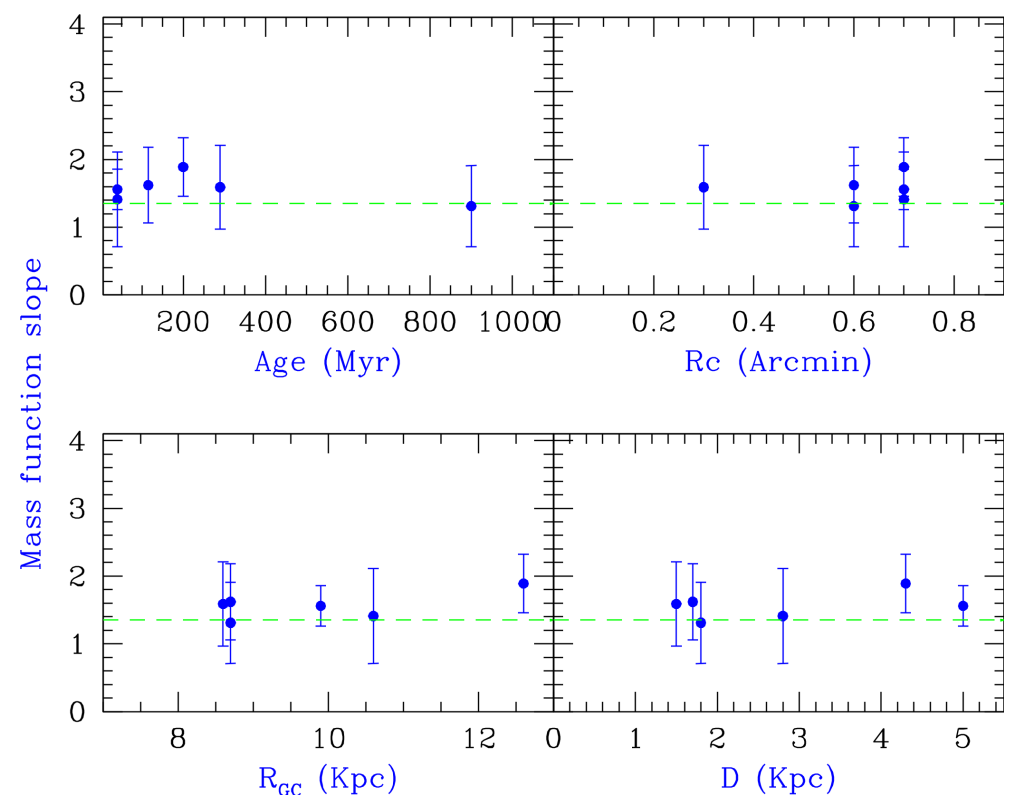

Figure 3: Dependence of the slope of the mass function with age, core radius $\left(R_{c}\right)$, galactocentric distance $\left(R_{G C}\right)$ and heliocentric distance $(D)$ of the clusters. The horizontal green line represents the slope of the Salpeter (1955). 


\section{Conclusions}

In this paper, we have studied the structure of our galaxy using open clusters in the second Galactic quadrant. Our study shows that up to a distance of $3.5 \mathrm{Kpc}$, the Galactic disc bends towards the southern hemisphere while it bends towards the northern hemisphere at larger distances. The distributions of the reddening with longitude and age show a decreasing trend. Our study shows that younger clusters have more reddening than older ones. We found that, the MF slope does not show any trend with clusters age, core radius, galactocentric distance and distance from the Sun.

\section{Acknowledgements}

The work is partly supported by the Department of Space, GOI. We are thankful to the ARIES staff for their assistance during observations and data reduction. This publication has made use of data from the $2 M A S S$, which is a joint project of the university of Massachusetts and the infrared processing and Analysis Center/California Institute of Technology, funded by the National Aeronautics and Space Administration and the National Science Foundation (NASA). This research has made use of WEBDA database.

\section{References}

Becker W. 1963, ZAp, 57, 117

Bisht D., Yadav R. K. S., Durgapal A. K. 2016, NewA, 42, 66

Bisht D., Yadav R. K. S., Durgapal A. K. 2017, NewA, 52, 55

Dias W. S., Alessi B. S., Moitinho A., Lepine J. R. D. 2002, A\&A, 389, 871

Digel S., de Geus E., Thaddeus P. 1994, ApJ, 422, 92

Digel S. W., Lyder D. A., Philbrick A. J., Puche D., Thaddeus P. 1996, ApJ, 458,561

Heyer M. H., Terebey S. 1998, ApJ, 502, 265

Kulkarni S. R., Heiles C., Blitz L. 1982, ApJ, 259, L63

Momany Y., Zaggia S., Gilmore G. et al. 2006, A\&A, 451, 515

Pandey A. K., Sharma S., Ogura K. 2006, MNRAS, 373, 255

Russeil D. 2003, A\&A, 397, 133

Salpeter E. E. 1955, ApJ, 121, 161

Solomon P. M., Rivolo A. R., Barrett J., Yahil A. 1987, ApJ, 319, 730

Subramaniam A., Bhatt B. C. 2007, MNRAS, 377, 829

Vallee J. P. 2005, AJ, 130, 569 